\title{
FATORES DETERMINANTES DA SATISFAÇÃO COM A EXPERIÊNCIA ACADÉMICA
}

\author{
SATISFACTION REGARDING ACADEMIC EXPERIENCE CAUSAL FACTOR
}

\author{
Adriana Silva Souza Rodrigues \\ pesquisa@fucape.br \\ Fucape Business School, Vitória/ES, BRASIL \\ Giuliana Bronzoni Liberato \\ gliberato@gmail.com \\ Fucape Business School, Vitória/ES, BRASIL
}

Recebido em 21.12.2015. Aprovado em 28.05.2016

Avaliado pelo sistema double blind review

DOI: http://dx.doi.org/10.12712/rpca.v10i2.703

\section{Resumo}

Este trabalho como objetivo verificar a satisfação dos estudantes de ensino superior com a experiência acadêmica e a sua relação com a vontade de concluir o curso. A metodologia utilizada foi pesquisa de natureza quantitativa, de corte transversal, realizada com estudantes em início de curso de engenharias em IES privadas das cidades de Belo Horizonte e Vitória. O questionário partiu de uma escala validada com três fatores: satisfação com o curso, satisfação com a instituição de ensino superior e oportunidades de desenvolvimento. Os resultados apontam que o fator satisfação com o curso foi o que apresentou maior significância estatística, as variáveis como corpo docente com domínio do conteúdo, estratégia de aula utilizada pelos professores, compromisso da instituição com a qualidade da formação do aluno, relacionamento com os professores, equipamentos modernos, empregabilidade potencial do curso apresentam importância na previsão do estudante em concluir o curso.

Palavras-chave: Experiência Acadêmica. Engenharia. Marketing Educacional.

\section{Abstract}

This paper aims at verifying third-level education students' satisfaction regarding academic experience and their relation with the will to conclude the course. The methodology used was a quantitative research, in crosssection, held with the freshmen engineering students at private IES in the cities of Belo Horizonte and Vitória. The questionnaire was based on scale validated in three factors: satisfaction with the course, satisfaction with the higher education institution and development opportunities. The results indicate that the satisfaction factor with the course itself was the one presenting higher statistical significance, the variables, such as, faculty with authority in the content, lesson strategy used by the Professors, institution commitment with the students 'quality formation, relationship with the Professors, modern equipment and potential employability of the course show relevance in the students 'prediction to finish the course.

Keywords: Academic experience. Engineering. Educational Marketing. 


\section{Introdução}

O cenário educacional brasileiro, em particular, o cenário das instituições de ensino superior (IES) tem passado por profundas mudanças nas últimas décadas. Cabe ressaltar que a qualidade dos serviços oferecidos pelas instituições de ensino e a satisfação dos discentes fazem parte destas alterações (GOMES; DAGOSTINI; CUNHA, 2013).

Apesar da expansão, um fato preocupante em relação a ela é que está ocorrendo uma facilidade de ingresso no ensino superior, ao passo que há pouca preocupação para que os estudantes sejam bem-sucedidos ao longo das suas experiências educacionais. Como resultado, a satisfação acadêmica e a qualidade do ensino podem ser comprometidas (ANTONELLI; COLAUTO; CUNHA， 2012; SCHLEICH; POLYDORO; SANTOS, 2006), sendo assim importante compreender a interação entre a instituição de ensino e o aluno ingressante, no que compete às mudanças provocadas a partir dessa interação (CARO; BOLARIN, 2011).

Assim, o potencial do marketing, como filosofia orientadora da sua gestão, pode trazer benefícios à organização, por meio da realização dos seus objetivos e do cumprimento da sua missão, e também pode beneficiar seu público-alvo, por meio de maior nível da satisfação (ALVES; MAINARDES; RAPOSO, 2010).

Diante deste contexto tem-se como objetivo geral verificar a satisfação dos estudantes de ensino superior com a experiência acadêmica e a sua relação com a vontade de concluir o curso.

Este estudo propõe contribuições distintas em relação ao trabalho de Schleich, Polydoro e Santos (2006), que propôs uma escala para investigar a satisfação do estudante de ensino superior abrangendo uma variedade de aspectos relacionados ao curso, à instituição e às oportunidades de desenvolvimento pessoal e profissional, bem como os aspectos relacionais envolvidos no contexto acadêmico. Partindo da escala de Schleich, Polydoro e Santos (2006) quanto às dimensões na escala de satisfação, foram introduzidos e retirados itens da escala original para realizar uma pesquisa com estudantes em início de curso de engenharias em IES privadas das cidades de Belo Horizonte e Vitória.
Pasquini et al. (2014) afirmam que há um nível crescente de insatisfação entre os alunos de ensino superior, que é imperceptível nos dois primeiros semestres. Este nível se torna perceptível apenas após o primeiro ano e aumenta no decorrer do curso e levando a desmotivação.

Apesar de muitas pesquisas sobre a natureza da satisfação, ainda não há uma definição clara e consistente sobre tal construto, havendo discussões teóricas ainda não suficientes sobre $\mathrm{O}$ tema (BENJAMIN; HOLLINGS, 1997). Também é relevante acentuar que a satisfação acadêmica é um fator que interfere diretamente na perpetuidade e no desenvolvimento da IES (GOMES; DAGOSTINI; CUNHA, 2013), sendo argumentos justificativos para a escolha do tema.

Nesse sentido, no Brasil, alguns estudos abordam diretamente a satisfação dos estudantes. Por exemplo, Bardagi, Lassance e Paradiso (2003) investigaram trajetórias acadêmicas, satisfação com a escolha profissional e expectativas quanto à orientação profissional com estudantes de semestres intermediários em 16 cursos universitários em uma capital brasileira da região sul. Scleich, Polydoro e Santos (2006) desenvolveram uma escala de satisfação com a experiência acadêmica com estudantes de uma IES particular do interior paulista. Já Gomes, Dagostini e Cunha (2013) avaliaram os determinantes da satisfação geral dos alunos matriculados no curso de Ciências Contábeis em uma faculdade do Sudoeste do Paraná. Antonielli, Colauto e Cunha (2012) estudaram as expectativas e satisfação dos alunos de ciências contábeis com relação às competências docentes. No exterior, destaca-se o estudo longitudinal de Caro e Bolarín (2011), que investigaram a satisfação dos alunos de 21 cursos de graduação e pós-graduação de engenharia, no primeiro e segundo ano. Schreiner (2009) estudou a conexão entre satisfação dos estudantes e retenção. Nath e Anderson (2007) analisaram os efeitos da técnica de discussão em sala de aula na satisfação dos estudantes.

Outros estudos abordam a satisfação dos estudantes como meio para se compreender as abordagens de marketing educacional que mais se adequam às necessidades das IES. Nesse sentido, Bergamo, Giuliani e Galli (2011) desenvolveram um Modelo de Lealdade e Retenção de Alunos para IES com base em diversas variáveis, como satisfação, qualidade 
percebida, integração social, integração acadêmica, comprometimento com atividades profissionais, comprometimento com atividades não-universitárias, dentre outras. Andrade e Araujo (2011) procuraram conhecer melhor o comportamento dos indivíduos na escolha por serviços em educação, realizando-se uma segmentação psicográfica em uma IES de uma capital da região nordeste.

No tocante aos cursos de Engenharia, pesquisas realizadas nos últimos dez anos apontam que esses cursos estão entre os que possuem maiores índices de evasão (BARBOSA; MEZZOMO; LORDER, 2011). Além disso, esse segmento profissional possui uma característica bastante peculiar. Segundo Lins et al. (2014), durante as décadas de 1980 e 1990, houve um crescimento econômico modesto, no qual projetos de infraestrutura foram reduzidos $\mathrm{e}$ os cursos de Engenharia tiveram pouca atratividade devido à instabilidade do mercado neste período. Quando a economia se aqueceu novamente a partir de 2000, o setor de tecnológico cresceu e a demanda por engenheiros voltou a aumentar, porém não havia no mercado oferta suficiente destes profissionais mais experientes. O resultado foi um déficit de engenheiros no mercado.

Assim, para o alcance do objetivo do presente estudo utilizou-se como metodologia uma pesquisa de natureza quantitativa, de corte transversal, realizada com estudantes em início de curso de engenharias em IES privadas das cidades de Belo Horizonte e Vitória. O questionário partiu de uma escala validada com três fatores: satisfação com o curso, satisfação com a instituição de ensino superior e oportunidades de desenvolvimento.

\section{Marketing Educacional}

O foco no consumidor é um dos princípios da gestão da qualidade e traz implicações não só na identificação dos clientes pela empresa, como também na implementação de mecanismos que detectem a satisfação, as necessidades e as expectativas do cliente (SARRICO; ROSA, 2014). Isso impacta no marketing educacional. Assim surge a necessidade de reconhecer o marketing como uma ferramenta de captação e retenção de estudantes para as IES privadas (ANDRADE; ARAUJO, 2011).
Diante desse cenário, a sobrevivência das IES depende de suas capacidades estratégicas para competirem e obterem resultados satisfatórios, o que vem sendo almejado por vários gestores educacionais (BERGAMO, 2008). Desta forma, as instituições privadas de ensino superior estão cada vez mais focadas em se tornarem altamente competitivas, por meio da valorização do mix de serviços oferecidos, com especial atenção à qualidade e à infraestrutura da instituição de ensino superior (MILAN et al., 2014).

As IES possuem condições favoráveis para saírem de um modelo de massa, no qual o intuito é oferecer o maior número de produtos, de forma mais padronizada possível, para um modelo segmentado, no qual os cursos seriam personalizados de acordo com a demanda do mercado, regiões de atuação, perfil de estudante, dentre outras características. Tal mudança possibilitaria às essas organizações buscarem um diferencial neste mercado (ANDRADE; ARAÚJO, 2011).

O marketing educacional surge a partir da oferta da IES focada na diversificação, visando estratégias específicas para cada um desses segmentos. Com um público com desejos variados, as IES focam em determinadas ações para surpreender as expectativas destes jovens, seja oferecendo melhores condições sobre localização e conforto na instituição, seja oferecendo facilidade de pagamento, visando atingir um público determinado como cliente (NUNES et al., 2008). Assim, as IES, tomando conhecimento de seus diferentes públicos alvo, podem estabelecer estratégias diferenciadas de sucesso (ANDRADE; ARAUJO, 2011).

Contudo, os programas pedagógicos das instituições oferecem uma atuação limitada para a gestão do marketing e, em muitos casos, as ações de marketing se limitam a estabelecer estratégias de captação de alunos para que o curso tenha viabilidade econômica e estratégias de mídia e promoções (NUNES et al., 2008).

Desta forma, o marketing educacional surge como uma ferramenta importante para que IES alcancem a satisfação de seus alunos (ANDRADE; ARAUJO, 2011). Ou seja, a satisfação dos discentes e suas expectativas são vistas pela instituição como um fator chave para avaliar a qualidade dos serviços prestados, inclusive para aquelas voltadas ao ensino 
(ANTONELLI; COLAUTO; CUNHA, 2012). Assim, o marketing educacional pode ajudar as IES a cumprir esse objetivo, uma vez que a satisfação é impactada pelo atendimento às expectativas e pela experiência educacional dos alunos. O aluno, no momento em que é inserido na universidade, forma um conceito sobre a universidade com base em suas informações iniciais disponíveis. Mais tarde, o aluno passa a comparar esse conceito com o ambiente e o curso.

Um ponto de partida para formular estratégias de marketing educacional bem-sucedidas é a compreensão dos atributos de satisfação do estudante com a experiência acadêmica para entender até que ponto isso impacta na vontade dos alunos em concluir um curso superior.

\section{Satisfação de estudante e atributos de satisfação de estudantes}

Mensurar satisfação é algo bastante complexo. Uma das dificuldades em realizar tal ato é a relatividade excessiva, por ser uma característica psicológica do indivíduo. O grau de satisfação também pode variar ao longo do tempo, uma vez que novas informações podem modificar o cenário pesquisado (SOUZA; ALVES; BUSS, 2008; BEBER, 1999).

Segundo Tontini e Walter (2011), é possível mensurar a satisfação dos estudantes em relação ao ensino superior. Eles afirmam que as necessidades do aluno devem ser identificadas permanentemente, tais como os fatores estruturais, administrativos e pedagógicos como forma de melhorar a qualidade do ensino superior e a capacidade de aprendizado do aluno.

Souza, Alves e Buss (2008) analisam a satisfação a partir dos fatores i) pessoais, relacionados ao discente e ii) institucionais, relacionados à experiência institucional. Os fatores que exercem influência positiva na satisfação são: amigos de grupo, identificação pessoal com o curso, mercado de trabalho propício, boa estrutura da grade curricular, atendimento das necessidades em geral dos alunos, habilidades e didática dos professores, material de apoio, percepção do ensino como proficiente. E os fatores que impactam negativamente na satisfação são: má organização da instituição, não atendimento às expectativas dos alunos e corpo docente indisponível para atender a demanda dos estudantes. Há ainda os que não apresentam influência considerável na satisfação: atividades extracurriculares ou atividades esportivas e sociais no campus.

No ambiente educacional, a qualidade do mix de serviços oferecidos pela IES é primordial a sua sobrevivência no mercado. A qualidade da grade do curso, a interação entre aluno e professor, além do envolvimento e a satisfação dos alunos com o curso, constituem atributos importantes para determinar o perfil e desempenho dos estudantes que mais tarde se formarão profissionais capacitados para o ambiente de trabalho (VENTURINI et al., 2008).

Quanto a satisfação dos alunos dos cursos de graduação com atributos relacionados exclusivamente a instituição de ensino, Mainardes e Domingues (2010) explicam que o ambiente universitário, clima no campus, vida no campus é o fator de maior satisfação entre os respondentes. Outro item relacionado a satisfação é a imagem que os alunos de uma forma geral têm da IES, pode-se concluir que o bom ambiente amplia a boa imagem da instituição entre os alunos. A qualidade da IES é fator relevante para satisfação, bem como o bom desempenho da coordenação e organização dos cursos, administração dos cursos, competência dos administradores e coordenadores. Os resultados que menos inferem sobre a satisfação referem-se a: atividades desportivas, culturais e associações de alunos; gerenciamento e solução de reclamações de alunos; ligações da IES com o exterior e intercâmbios com o estrangeiro; preço, condições de pagamento e serviços financeiros.

Aitken (1982) assume que a retenção dos alunos em uma instituição específica acontece em função da satisfação dos alunos e a satisfação pode ser medida pela: satisfação com o ambiente, o próprio desempenho acadêmico, o nível de envolvimento em atividades extracurriculares, e alguns fatores externos.

O estudo de Silva et al. (2012) identificou aspectos que influenciam positivamente a satisfação dos estudantes: os amigos; a identificação do indivíduo com o programa do curso; aspectos externos tais como as condições favoráveis para que ele alcance um emprego após se formar; respostas que satisfaçam suas necessidades e expectativas em geral e boa estrutura do curso; habilidades, conhecimentos, estratégias; e, interação entre os alunos e os professores. Já os aspectos negativos são: o despreparo e pouco compromisso dos professores; a decepção em não 
ter as expectativas correspondidas; e dificuldade/ incapacidade para responder as necessidades do estudante.

Quevedo-Silva et al. (2012) salientam um fator determinante importante: o ambiente social. É importante analisar as interações entre professor e estudante quando o assunto é insatisfação. O comportamento desinteressado de alguns colegas de classe com o curso pode impactar o aprendizado dos demais, de forma a gerar receio nos alunos interessados de sofrer punições por parte dos docentes.

Alguns estudos, ao longo do tempo, propuseram diferentes atributos para a satisfação de estudantes, conforme pode ser observado no Quadro 1.

Quadro 1: Atributos considerados para a satisfação de estudantes

\begin{tabular}{|c|c|}
\hline ATRIBUTOS & TEORIA \\
\hline $\begin{array}{l}\text { Recursos e equipamentos } \\
\text { disponíveis pela instituição }\end{array}$ & Clayson e Haley (1990) \\
\hline Sala de estudo complementar & Paswan e Young (2002) \\
\hline $\begin{array}{l}\text { Relevância do conteúdo das } \\
\text { matérias }\end{array}$ & Paswan e Young (2002) \\
\hline $\begin{array}{l}\text { Avaliação proposta pelos } \\
\text { professores e Infraestrutura das } \\
\text { salas de aula }\end{array}$ & Paswan e Young (2002) \\
\hline Facilidade no contato via telefone & Pinheiro (2003) \\
\hline Atendimento a reclamações & Pinheiro (2003) \\
\hline $\begin{array}{l}\text { Reconhecimento do curso perante } \\
\text { a sociedade }\end{array}$ & $\begin{array}{l}\text { Hides, Davies e Jackson } \\
(2004)\end{array}$ \\
\hline $\begin{array}{l}\text { Relacionamento com os } \\
\text { professores }\end{array}$ & Kanan e Baker (2006) \\
\hline $\begin{array}{l}\text { Atendimento personalizado aos } \\
\text { alunos }\end{array}$ & Kanan e Baker (2006) \\
\hline $\begin{array}{l}\text { Empregabilidade potencial do } \\
\text { curso }\end{array}$ & Venturini et al. (2008) \\
\hline $\begin{array}{l}\text { Compromisso da instituição } \\
\text { de ensino com a qualidade da } \\
\text { formação do aluno }\end{array}$ & Venturini et al. (2008) \\
\hline Currículo do curso & \begin{tabular}{|llll}
$\begin{array}{l}\text { Souza, Alves e } \\
(2008)\end{array}$ & & \\
\end{tabular} \\
\hline $\begin{array}{l}\text { Atividades extracurriculares } \\
\text { oferecidas pela instituição }\end{array}$ & $\begin{array}{l}\text { Souza, Alves e Buss } \\
(2008) \text {, Aitken (1982) } \\
\end{array}$ \\
\hline $\begin{array}{l}\text { Facilidade da instituição em atender } \\
\text { questões pessoais }\end{array}$ & \begin{tabular}{|l} 
Quevedo-Silva et al. \\
$(2012)$
\end{tabular} \\
\hline $\begin{array}{l}\text { Atendimento prestado pelos } \\
\text { funcionários da secretaria }\end{array}$ & \begin{tabular}{|l}
$\begin{array}{l}\text { Quevedo-Silva et al. } \\
(2012)\end{array}$ \\
\end{tabular} \\
\hline Localização da instituição & Schreiner (2009) \\
\hline
\end{tabular}

\begin{tabular}{|l|l|}
\hline $\begin{array}{l}\text { Relacionamento com os colegas de } \\
\text { sala }\end{array}$ & Schreiner (2009) \\
\hline Preço das mensalidades & Silva et al. (2012) \\
\hline $\begin{array}{l}\text { Programas ou serviços de apoio } \\
\text { oferecidos pela instituição }\end{array}$ & Silva et al. (2012) \\
\hline O próprio desempenho acadêmico & Aitken (1982) \\
\hline Satisfação com o ambiente & Aitken (1982) \\
\hline Fatores externos & Aitken (1982) \\
\hline
\end{tabular}

Fonte: Adaptado de Schleich, Polydoro e Santos (2006) e ampliado pelos autores.

\section{Metodologia}

Para cumprir o objetivo proposto para essa pesquisa, foi conduzido um estudo de natureza quantitativa e de corte transversal. A população desse estudo compreendeu os estudantes de IES privadas de cursos de Engenharia e a amostra incluiu os estudantes de Engenharia dos anos iniciais de IES privadas das cidades de Vitória e Belo Horizonte. A escolha por essas cidades foi aleatória. Os questionários foram aplicados no período que compreende fevereiro e março de 2014.

Com o objetivo de verificar a satisfação dos estudantes de ensino superior com a experiência acadêmica e a sua relação com a vontade de concluir o curso, foi aplicado um questionário adaptado a partir da escala de Schleich, Polydoro e Santos (2006), apresentada no Quadro 2. Foram feitos alguns ajustes à escala original.

Quadro 2: Escala de satisfação com a experiência acadêmica (ESEA)

\begin{tabular}{|cl}
\hline \multicolumn{2}{c}{ Itens da escala de dimensão } \\
\hline Satisfação com o Curso \\
$\checkmark$ & Relacionamento com os professores \\
$\checkmark$ & Relacionamento com os colegas do curso \\
$\checkmark$ & Adequação entre o envolvimento pessoal no curso e \\
$\checkmark$ & o desempenho acadêmico obtido \\
$\checkmark$ & Interesse dos professores em atender os estudantes \\
$\checkmark$ & Conhecimento dos professores sobre o conteúdo \\
$\checkmark$ & Reconhecimento por parte dos professores do meu \\
$\checkmark$ & formprimeção \\
$\checkmark$ & Avaliação proposta pelos professores \\
$\checkmark$ & Relevânciancia do conteúdo das disciplinas
\end{tabular}


$\checkmark \quad$ Disponibilidade dos professores em atender os alunos fora da sala de aula

$\checkmark$ Adequação do conteúdo para formação

$\checkmark$ Adequação entre as tarefas exigidas no curso e o tempo estabelecido pelos professores para realização

Oportunidade de desenvolvimento

$\checkmark$ Diversidade das atividades extracurriculares oferecidas pela instituição

$\checkmark$ Curriculum do curso

$\checkmark$ Eventos sociais oferecidos pela instituição

$\checkmark$ Envolvimento pessoal nas atividades do curso

$\checkmark$ Programas ou serviços de apoio aos estudantes oferecidos pela instituição

$\checkmark$ Condições oferecidas para o meu desenvolvimento profissional

$\checkmark$ Condições para ingresso na área profissional de formação

$\checkmark$ Programa de apoio financeiro oferecido pela instituição

$\checkmark$ Oportunidade de desenvolvimento pessoal oferecida pela instituição

$\checkmark$ Adequação entre o meu investimento financeiro para custear os estudos e a formação recebida

Satisfação com a Instituição

$\checkmark$ Recursos e equipamentos audiovisuais disponíveis na instituição

$\checkmark$ Atendimento e clareza das informações oferecidas pelos funcionários da secretaria

$\checkmark$ Equipamentos e softwares oferecidos pelo laboratório de informática

$\checkmark$ Atendimento e clareza das informações oferecidas pelos funcionários da biblioteca

$\checkmark$ Acervo disponível na biblioteca

$\checkmark$ Segurança oferecida pela instituição

$\checkmark$ Infraestrutura física das salas de aula

$\checkmark$ Infraestrutura física da instituição

$\checkmark$ Limpeza da instituição

$\checkmark$ Serviços oferecidos pela biblioteca

$\checkmark$ Conformidade das instalações da instituição

$\checkmark$ Localização dos diferentes setores que compõem a instituição

Fonte: Adaptado de Schleich; Polydoro; Santos (2006, p. 17)

Partindo do princípio da multidimensionalidade da mensuração da satisfação, e com o intuito de evitar questões múltiplas (HAIR Jr. et al., 2005), os itens da pesquisa de Schleich, Polydoro e Santos (2006) - "condições oferecidas para o meu desenvolvimento profissional" e "condições para ingresso na área profissional de formação", foram reduzidos a uma única pergunta no questionário dessa pesquisa: "empregabilidade potencial do curso". De forma análoga, os itens "atendimento e clareza das informações oferecidas pelos funcionários da secretaria" e "atendimento e clareza das informações oferecidas pelos funcionários da biblioteca", foram reduzidos a um único item: "atendimento personalizado aos alunos".

Da escala original do trabalho de Schleich, Polydoro e Santos (2006) com 32 itens, 18 itens foram mantidos; 14 itens foram excluídos; 04 itens foram reduzidos a 02 itens, conforme explicado acima, e 08 novos itens foram incluídos. Por último, foram feitos ajustes na redação com o intuito de melhor adequação do texto.

Assim, o questionário final incluiu vinte e oito itens mensurados pela escala Likert de sete pontos, sendo 1 [Não influencia] e 7 [influencia muito], além da inclusão da variável dependente "conclusão do curso", conforme Quadro 3.

Quadro 3: Questionário de satisfação com a experiência acadêmica aplicado na pesquisa

\begin{tabular}{|c|c|c|c|c|c|c|c|c|}
\hline \multicolumn{2}{|r|}{ Itens do questionário } & \multicolumn{3}{|c|}{$\begin{array}{c}\text { Não } \\
\text { influencia }\end{array}$} & \multicolumn{4}{|c|}{$\begin{array}{c}\text { Influencia } \\
\text { muito }\end{array}$} \\
\hline 1 & $\begin{array}{l}\text { Relacionamento com os } \\
\text { professores }\end{array}$ & 1 & 2 & 3 & 4 & 5 & 6 & 7 \\
\hline 2 & $\begin{array}{l}\text { Relacionamento com os colegas } \\
\text { de sala }\end{array}$ & 1 & 2 & 3 & 4 & 5 & 6 & 7 \\
\hline 3 & $\begin{array}{l}\text { Interesse do corpo docente em } \\
\text { atender os alunos durante a aula }\end{array}$ & 1 & 2 & 3 & 4 & 5 & 6 & 7 \\
\hline 4 & $\begin{array}{l}\text { Corpo docente com domínio do } \\
\text { conteúdo }\end{array}$ & 1 & 2 & 3 & 4 & 5 & 6 & 7 \\
\hline 5 & $\begin{array}{l}\text { Compromisso da instituição } \\
\text { de ensino com a qualidade da } \\
\text { formação do aluno }\end{array}$ & 1 & 2 & 3 & 4 & 5 & 6 & 7 \\
\hline 6 & $\begin{array}{l}\text { Avaliação proposta pelos } \\
\text { professores }\end{array}$ & 1 & 2 & 3 & 4 & 5 & 6 & 7 \\
\hline 7 & $\begin{array}{l}\text { Estratégia de aula utilizada pelos } \\
\text { professores }\end{array}$ & 1 & 2 & 3 & 4 & 5 & 6 & 7 \\
\hline 8 & $\begin{array}{l}\text { Relevância do conteúdo das } \\
\text { matérias }\end{array}$ & 1 & 2 & 3 & 4 & 5 & 6 & 7 \\
\hline 9 & $\begin{array}{l}\text { Atividades extracurriculares } \\
\text { oferecidas pela instituição }\end{array}$ & 1 & 2 & 3 & 4 & 5 & 6 & 7 \\
\hline 10 & Curriculum do curso & 1 & 2 & 3 & 4 & 5 & 6 & 7 \\
\hline 11 & $\begin{array}{l}\text { Eventos sociais oferecidos pela } \\
\text { instituição }\end{array}$ & 1 & 2 & 3 & 4 & 5 & 6 & 7 \\
\hline 12 & $\begin{array}{l}\text { Programas ou serviços de apoio } \\
\text { oferecidos pela instituição }\end{array}$ & 1 & 2 & 3 & 4 & 5 & 6 & 7 \\
\hline 13 & $\begin{array}{l}\text { Recursos e equipamentos } \\
\text { disponíveis pela instituição }\end{array}$ & 1 & 2 & 3 & 4 & 5 & 6 & 7 \\
\hline 14 & $\begin{array}{l}\text { Atendimento prestado pelos } \\
\text { funcionários da secretaria }\end{array}$ & 1 & 2 & 3 & 4 & 5 & 6 & 7 \\
\hline 15 & Biblioteca & 1 & 2 & 3 & 4 & 5 & 6 & 7 \\
\hline 16 & Segurança na instituição & 1 & 2 & 3 & 4 & 5 & 6 & 7 \\
\hline 17 & Infraestrutura das salas de aula & 1 & 2 & 3 & 4 & 5 & 6 & 7 \\
\hline 18 & Limpeza da instituição & 1 & 2 & 3 & 4 & 5 & 6 & 7 \\
\hline 19 & Localização da instituição & 1 & 2 & 3 & 4 & 5 & 6 & 7 \\
\hline 20 & $\begin{array}{l}\text { Atendimento personalizado aos } \\
\text { alunos }\end{array}$ & 1 & 2 & 3 & 4 & 5 & 6 & 7 \\
\hline 21 & Preço das mensalidades & 1 & 2 & 3 & 4 & 5 & 6 & 7 \\
\hline 22 & $\begin{array}{l}\text { Reconhecimento do curso } \\
\text { perante à sociedade }\end{array}$ & 1 & 2 & 3 & 4 & 5 & 6 & 7 \\
\hline 23 & Facilidade no contato via telefone & 1 & 2 & 3 & 4 & 5 & 6 & 7 \\
\hline
\end{tabular}




\begin{tabular}{|c|c|c|c|c|c|c|c|c|c|c|c|}
\hline 24 & \multicolumn{4}{|c|}{$\begin{array}{l}\text { Flexibilidade da instituição em } \\
\text { atender questões pessoais }\end{array}$} & 1 & 2 & 3 & 4 & 5 & 6 & 7 \\
\hline 25 & \multicolumn{4}{|c|}{$\begin{array}{l}\text { Empregabilidade potencial do } \\
\text { curso }\end{array}$} & 1 & 2 & 3 & 4 & 5 & 6 & 7 \\
\hline 26 & \multicolumn{4}{|c|}{$\begin{array}{l}\text { Existência de equipamentos } \\
\text { modernos }\end{array}$} & 1 & 2 & 3 & 4 & 5 & 6 & 7 \\
\hline 27 & \multicolumn{4}{|c|}{ Atendimento a reclamações } & 1 & 2 & 3 & 4 & 5 & 6 & 7 \\
\hline 28 & \multicolumn{4}{|c|}{ Sala de estudo complementar } & 1 & 2 & 3 & 4 & 5 & 6 & 7 \\
\hline \multicolumn{12}{|c|}{$\begin{array}{l}\text { Entre } 1 \text { e } 7 \text {, Indique uma nota que represente a sua vontade de fazer } \\
\text { o curso completamente: }\end{array}$} \\
\hline \multicolumn{12}{|c|}{$\begin{array}{l}\text { Com certeza não irei concluir o curso } \\
\text { Com certeza irei concluir o curso }\end{array}$} \\
\hline \multicolumn{2}{|c|}{1} & 2 & 3 & 4 & 5 & \multicolumn{2}{|c|}{6} & \multicolumn{3}{|c|}{7} & \\
\hline
\end{tabular}

Fonte: Elaboração própria. Nota: itens de 1 a $18=$ itens mantidos; itens 19, 21-24 26-28 = itens adicionados; itens 20 e $25=$ itens transformados.

Como os dados são ordenados, foi usada uma regressão múltipla para verificar se há uma relação linear entre a satisfação com a experiência acadêmica (satisfação com o curso, satisfação com a IES e oportunidades de desenvolvimento) e a vontade de concluir o curso superior de Engenharia nas cidades de Vitória e Belo Horizonte. Com o intuito de usar os três fatores (satisfação com o curso, satisfação com a IES e oportunidades de desenvolvimento), foram calculados os escores somados para representar esses mesmos constructos.

A seguir, o modelo de regressão e as explicações das variáveis.

Conclusão do curso $=\mathrm{b}_{\mathrm{o}}+\mathrm{b}_{1^{*}}$ satisfação com o
curso $+\mathrm{b}_{2^{*}}$ satisfação com a IES $+\mathrm{b}_{3^{*}}$ oportunidades de
desenvolvimento $+\mathrm{u}$

Onde: Conclusão do curso $=$ representa as respostas dos alunos de 1 a 7 (1 = com certeza não irei concluir o curso e $7=$ com certeza irei concluir o curso para a pergunta sobre a vontade de fazer o curso completamente).

- Satisfação com o curso representa o fator composto pelas perguntas: Corpo docente com domínio do conteúdo; Estratégia de aula utilizada pelos professores; Empregabilidade potencial do curso; Interesse do corpo docente em atender os alunos durante a aula; Compromisso da instituição de ensino com a qualidade da formação do aluno; Avaliação proposta pelos professores; Existência de equipamentos modernos; Relacionamento com os professores; Relevância do conteúdo das matérias; Recursos e equipamentos disponíveis pela instituição; Atendimento personalizado aos alunos e Atendimento a reclamações.

- Satisfação com a IES = representa o fator composto pelas perguntas: Limpeza da instituição; Biblioteca; Segurança na instituição; Localização da instituição; Infraestrutura das salas de aula; Facilidade no contato via telefone e Atendimento prestado pelos funcionários da secretaria.

- Oportunidades de desenvolvimento = representa o fator composto pelas perguntas: Eventos sociais oferecidos pela instituição; Atividades extracurriculares oferecidas pela instituição; Programas ou serviços de apoio oferecidos pela instituição; Facilidade da instituição em atender questões pessoais e Currículo do curso.

\section{Tratamento estatístico dos dados}

Quanto ao tratamento estatístico, os dados coletados junto a 86 alunos de Vitória e 56 de Belo Horizonte foram cadastrados e codificados em planilha eletrônica Excel, para posterior tratamento por meio do software SPSS e Eviews.

Ações reparadoras de dados não foram necessárias. As suposições de normalidade foram verificadas por meio de análises gráficas complementadas pelo teste estatístico de Kolmogorov-Smirnov, que mostrou resultados significativos $(0,000)$ ao nível de $5 \%$ $(<0,05)$ para todas as variáveis. As suposições de multicolinearidade e homocesdasticidade também foram atendidas.

Verificados tais pressupostos, a etapa seguinte consistiu da análise da validade e confiabilidade da escala utilizada, por meio da análise fatorial exploratória, uma vez que a escala original adotada por Schleich, Polydoro e Santos (2006) foi ajustada, conforme explicado anteriormente.

A adequação da análise fatorial foi verificada pelos 
testes de Kaiser-Meyer-Olkin (KMO) e de Esfericidade de Bartlett, mostrados na Tabela 1, que examinam a presença de correlações entre as variáveis (HAIR Jr. et al., 2005). O teste KMO de 0,881 apresenta valores normalizados (entre 0 e 1,0), indicando um grau adequado de ajuste à análise fatorial, ou seja, que esse método é adequado à análise fatorial como tratamento dos dados dessa pesquisa (HAIR Jr. et al., 2005). Já o Teste de Esfericidade de Bartlett mostrouse significante ao nível de $5 \%(<0,05)$, o que permite mais uma vez admitir a possibilidade e adequação do método de análise fatorial para o tratamento dos dados.

Tabela 1: Teste de Esfericidade de Bartlett

\begin{tabular}{|c|c|c|}
\hline \multicolumn{3}{|c|}{ KMO and Bartlett's Test } \\
\hline \multirow{4}{*}{$\begin{array}{l}\text { Kaiser-Meyer-Ol } \\
\text { Adequacy. } \\
\text { Bartlett's Test of } \\
\text { Sphericity }\end{array}$} & re of Sampling & ,881 \\
\hline & Approx. Chi-Square & 2103,656 \\
\hline & df & 378 \\
\hline & Sig. &, 000 \\
\hline
\end{tabular}

Fonte: Dados da pesquisa

A análise fatorial foi feita por extração de fatores principais com rotação varimax. Nesse caso, foram extraídos três fatores baseados no critério a priori, que se justifica por estar repetindo o estudo de outros pesquisadores (SCHEILCH; POLYDORO; SANTOS, 2006) e desejar extrair o mesmo número de fatores anteriormente encontrados (HAIR Jr. et al., 2005), ou seja, encontrar três fatores - satisfação com o curso, oportunidades de desenvolvimento e satisfação com a IES.

Ao avaliar a matriz fatorial na primeira análise realizada, as cargas fatoriais das variáveis "reconhecimento do curso perante a sociedade" $(0,40)$, "relacionamento com os colegas de sala" (0,39), "preço das mensalidades" $(0,31)$ e "sala de estudo complementar" $(0,40)$ ficaram abaixo das orientações recomendadas por (HAIR Jr. et al., 2005). Para esses autores, uma carga estatisticamente significante para o tamanho dessa amostra fica em torno de 0,45. Ademais, essas variáveis apresentaram comunalidades de $0,23,0,25$,
0,16 e 0,40 , respectivamente, muito abaixo do valor mínimo aceitável de 0,5.

Por isso, essas variáveis foram removidas e uma nova análise fatorial foi realizada. A Tabela 2 apresenta os três fatores com carga fatorial acima de 0,45: satisfação com o curso (12 itens); satisfação com a IES (sete itens); oportunidades de desenvolvimento (cinco itens).

Tabela 2: Matriz de Componente Rotativa

\begin{tabular}{|c|c|c|c|c|}
\hline \multirow{2}{*}{ Fatores } & \multirow{2}{*}{ Variáveis } & \multicolumn{3}{|c|}{ Componentes } \\
\hline & & 1 & 2 & 3 \\
\hline \multirow{12}{*}{$\begin{array}{l}\text { Satisfação com o } \\
\text { curso }\end{array}$} & $\begin{array}{l}\text { Corpo docente } \\
\text { com domínio do } \\
\text { conteúdo }\end{array}$ & ,796 & & \\
\hline & $\begin{array}{l}\text { Estratégia de aula } \\
\text { utilizada pelos } \\
\text { professores }\end{array}$ & ,711 & & \\
\hline & $\begin{array}{l}\text { Empregabilidade } \\
\text { potencial do curso }\end{array}$ & ,691 & & \\
\hline & $\begin{array}{l}\text { Interesse do corpo } \\
\text { docente em atender } \\
\text { os alunos durante } \\
\text { a aula }\end{array}$ & ,652 & & \\
\hline & $\begin{array}{l}\text { Compromisso da } \\
\text { instituição de ensino } \\
\text { com a qualidade da } \\
\text { formação do aluno }\end{array}$ & ,651 & & \\
\hline & $\begin{array}{l}\text { Avaliação proposta } \\
\text { pelos professores }\end{array}$ & ,641 & & \\
\hline & $\begin{array}{l}\text { Existência de } \\
\text { equipamentos } \\
\text { modernos }\end{array}$ & ,622 & & \\
\hline & $\begin{array}{l}\text { Relacionamento } \\
\text { com os professores }\end{array}$ & ,621 & & \\
\hline & $\begin{array}{l}\text { Relevância do } \\
\text { conteúdo das } \\
\text { matérias }\end{array}$ & 601 & & \\
\hline & $\begin{array}{l}\text { Recursos e } \\
\text { equipamentos } \\
\text { disponíveis pela } \\
\text { instituição }\end{array}$ &, 598 & & \\
\hline & $\begin{array}{l}\text { Atendimento } \\
\text { personalizado aos } \\
\text { alunos }\end{array}$ & ,490 & & \\
\hline & $\begin{array}{l}\text { Atendimento a } \\
\text { reclamações }\end{array}$ & ,489 & & \\
\hline
\end{tabular}




\begin{tabular}{|c|c|c|c|}
\hline \multirow{7}{*}{$\begin{array}{l}\text { Satisfação com } \\
\text { a IES }\end{array}$} & $\begin{array}{l}\text { Limpeza da } \\
\text { instituição }\end{array}$ &, 787 & \\
\hline & Biblioteca & ,712 & \\
\hline & $\begin{array}{l}\text { Segurança na } \\
\text { instituição }\end{array}$ & ,697 & \\
\hline & $\begin{array}{l}\text { Localização da } \\
\text { instituição }\end{array}$ &, 595 & \\
\hline & $\begin{array}{l}\text { Infraestrutura das } \\
\text { salas de aula }\end{array}$ &, 547 & \\
\hline & $\begin{array}{l}\text { Facilidade no } \\
\text { contato via telefone }\end{array}$ &, 546 & \\
\hline & $\begin{array}{l}\text { Atendimento } \\
\text { prestado pelos } \\
\text { funcionários da } \\
\text { secretaria }\end{array}$ & ,493 & \\
\hline \multirow{5}{*}{$\begin{array}{l}\text { Oportunidades de } \\
\text { desenvolvimento }\end{array}$} & $\begin{array}{l}\text { Eventos sociais } \\
\text { oferecidos pela } \\
\text { instituição }\end{array}$ & & ,811 \\
\hline & $\begin{array}{l}\text { Atividades } \\
\text { extracurriculares } \\
\text { oferecidas pela } \\
\text { instituição }\end{array}$ & & ,757 \\
\hline & $\begin{array}{l}\text { Programas ou } \\
\text { serviços de apoio } \\
\text { oferecidos pela } \\
\text { instituição }\end{array}$ & &, 735 \\
\hline & $\begin{array}{l}\text { Facilidade da } \\
\text { instituição em } \\
\text { atender questões } \\
\text { pessoais }\end{array}$ & &, 503 \\
\hline & Currículo do curso & & ,497 \\
\hline
\end{tabular}

Fonte: Dados da pesquisa.

A análise de confiabilidade dessa escala foi feita pelo Alfa de Cronbach, cuja variação é de 0,80 , ou seja, os três fatores podem ser combinados para mensurar a satisfação com a experiência acadêmica. Para a análise dos dados, foram utilizadas análises de médias e regressão linear múltipla em que conclusão do curso é a variável dependente e os fatores satisfação com o curso, satisfação com a IES e oportunidades de desenvolvimento são as variáveis independentes.

\section{Análise e discussão dos resultados}

A amostra é composta em sua maioria pelo gênero masculino, cerca de $65 \%$ e o curso que apresentou maior número de estudantes foi o de Engenharia Civil, $53 \%$. Já a faixa etária dos entrevistados concentra- se entre 16 a 25 anos (72\%). Cerca de 90\% dos estudantes entrevistados são solteiros e não possuem filhos e aproximadamente $50 \%$ dos alunos ainda não trabalham. Entre aqueles que exercem atividade remunerada, $40 \%$ estão trabalhando na iniciativa privada e $8 \%$ no setor público. Quanto às funções, $9 \%$ estão ocupando cargos de gestão - diretoria, gerência ou supervisão e cerca de $32 \%$ ainda ocupam cargos operacionais. Finalmente, quanto à localização das IES, Vitória corresponde a $60 \%$ dos respondentes e Belo Horizonte a $40 \%$.

A tabela 3 evidencia os resultados da regressão para as duas cidades pesquisadas. Por ela é possível afirmar que todos os fatores pesquisados (satisfação com o curso, satisfação com a IES e oportunidades de desenvolvimento) influenciam, de alguma forma, a satisfação com a experiência acadêmica e, portanto, na vontade de concluir o curso pelos estudantes do curso superior de Engenharia nos anos iniciais tanto em Vitória quanto em Belo Horizonte. Todas as médias para os fatores pesquisados estão acima do ponto intermediário $(4,0)$ da escala de sete pontos, representando, portanto, percepções positivas para os atributos.

Tabela 3: Médias Entre as Capitais de Belo Horizonte e Vitória

\begin{tabular}{llllc}
\hline Local IES & & Média & $\begin{array}{l}\text { Desvio } \\
\text { Padrão }\end{array}$ & N \\
\hline & $\begin{array}{l}\text { Conclusão de } \\
\text { curso }\end{array}$ & 6,670 &, 710 & 86 \\
Vitória & $\begin{array}{l}\text { Satisfação com o } \\
\text { curso }\end{array}$ & 5,462 & 1,063 & 86 \\
& $\begin{array}{l}\text { Oportunidades de } \\
\text { desenvolvimento }\end{array}$ & 4,727 & 1,306 & 86 \\
& $\begin{array}{l}\text { Satisfação com } \\
\text { a IES }\end{array}$ & 4,976 & 1,235 & 86 \\
\hline \multirow{5}{*}{$\begin{array}{l}\text { Conclusão de } \\
\text { curso }\end{array}$} & 6,540 &, 894 & 56 \\
Selo & $\begin{array}{l}\text { Satisfação com o } \\
\text { curso }\end{array}$ & 5,202 & 1,046 & 56 \\
Horizonte & $\begin{array}{l}\text { Oportunidades de } \\
\text { desenvolvimento }\end{array}$ & 4,907 & 1,001 & 56 \\
& $\begin{array}{l}\text { Satisfação com } \\
\text { a IES }\end{array}$ & 5,035 &, 9251 & 56 \\
\hline
\end{tabular}

Fonte: Elaborada pelos autores 
Os alunos de ambas as capitais foram coerentes em suas opiniões acerca da importância e vontade de concluírem o curso de Engenharia em suas IES. Isso pode ser medido pela variável dependente (conclusão do curso), que apresenta a média mais alta para ambas as cidades, atingindo 6,67 para Vitória e 6,04 para Belo Horizonte. O desvio-padrão para essa variável é de 0,710 para Vitória e de 0,894 para Belo Horizonte.

Esse resultado está alinhado com os estudos de alguns autores. Schleich, Polydoro e Santos (2006) afirmam que a importância em se concluir o curso, se dá uma vez que as contribuições das IES são primordiais para o desenvolvimento do discente, ao oferecer experiência acadêmica e compartilhamento de ideias, todavia esta experiência acadêmica depende do envolvimento dos estudantes com os recursos oferecidos pela instituição de ensino superior.

Já Lins et al (2014) fizeram um retrospecto da história econômica brasileira para justificar o crescimento da demanda por mão de obra qualificada de engenheiros e afirmam que a economia brasileira, por volta do ano 2000, apresentou um crescimento médio anual de 4,4\%. Isso implicou em mais investimentos em desenvolvimento tecnológico, em inovação e na contratação de mão de obra qualificada de engenheiros. Quando se analisa as taxas de desemprego pelos censos dos anos 2000 e 2010, conclui-se que a taxa de desemprego entre os engenheiros recém-formados caiu 2 pontos percentuais, reduzindo de $4 \%$ para $2 \%$.

Freitas, Dornellas e Belhot (2006) salientam a característica dinâmica e maleável do mercado e concluem que o engenheiro de produção deve se preparar para se inserir neste mercado, como profissional polivalente, e dizem que um erro considerado no ensino do curso de Engenharia é se orientar basicamente por exigências momentâneas do mercado, sem preparar o estudante para novos desafios econômicos e sociais que ele poderá enfrentar.

A média de satisfação com o curso dos alunos de Vitória $(5,46)$ é um pouco superior comparado com a média para os estudantes de Belo Horizonte $(5,20)$. Isso indica que os alunos de Vitória têm maior preocupação com o domínio do conteúdo pelo corpo docente, com as estratégias utilizadas em sala pelos professores, com a empregabilidade proporcionada pelo curso e com o compromisso da IES com a formação do aluno, entre outros fatores relacionados ao curso. Já o nível de satisfação com a IES é similar entre os alunos de ambas as capitais - médias de 4,9 para Vitória e de 5,03 para Belo Horizonte, indicando que quando se trata da limpeza e segurança da instituição, da robustez da biblioteca, da localização e infraestrutura as respostas seguem mesmo caminho. Entretanto, o fator satisfação com a IES na cidade de Belo Horizonte $(0,925)$ está dentro de um desviopadrão $(<1,0)$, mostrando mais coerência nas respostas do que os alunos de Vitória $(1,235)$.

Embora o fator oportunidades de desenvolvimento apresente a mais baixa média para ambas as cidades, atingindo 4,72 para Vitória e 4,90 para Belo Horizonte, os alunos da capital mineira estão um pouco mais satisfeitos com as oportunidades de desenvolvimento oferecidas pela IES. Ou seja, estão um pouco mais satisfeitos com os eventos sociais, atividades extracurriculares, programas ou serviços de apoio oferecidos pela instituição pelos estudantes, além da flexibilidade da instituição em atender questões pessoais e curriculum do curso. Também é importante relatar que o desvio-padrão $(1,306)$ para o fator oportunidades de desenvolvimento na cidade de Vitória é o mais alto, ou seja, há variabilidade de respostas quanto ao impacto desse fator na satisfação com a experiência acadêmica na IES dessa cidade.

\section{Análise de regressão múltipla para a cidade de vitória}

De acordo com os resultados mostrados na Tabela 4, há evidências que os atributos mais valorizados pelos estudantes de Engenharia da IES privada da cidade de Vitória abrangem o fator satisfação com o curso. Quanto mais satisfeitos os alunos estiverem com o curso, mais vontade eles terão de concluir o curso de Engenharia nessa cidade. Isso é demonstrado pelo regressor b1 $(0,044775$; $\mathrm{p}$-value $=0,0085)$ que apresenta significância estatística, ou seja, há evidências de relação positiva entre a satisfação com o curso e a vontade de concluir o curso de Engenharia para a cidade de Vitória. Os demais fatores - satisfação com a IES e oportunidades de desenvolvimento - não são estaticamente significantes. $O$ desvio padrão de 0,01701 é baixo, contribuindo para a confiabilidade desse coeficiente de regressão. 
Tabela 4: Modelo de Regressão para Vitória

\begin{tabular}{|c|c|c|c|c|}
\hline \multicolumn{5}{|c|}{ Variável dependente: CONCLUSÃO DO CURSO } \\
\hline \multicolumn{5}{|c|}{ Método: QML - Normal Count (Quadratic hill climbing) } \\
\hline \multicolumn{5}{|l|}{ Número de Observações : 86} \\
\hline \multicolumn{5}{|l|}{ Parâmetro usado na estimação QML: 1} \\
\hline \multicolumn{5}{|c|}{ Convergence achieved after 4 iterations } \\
\hline \multicolumn{5}{|c|}{ QML (Huber/White) standard errors \& covariance } \\
\hline & Coefficient & td. Error & z-Statistic & Prob. \\
\hline$\beta 0$ & 1,649377 & 0,094372 & 17,47743 & 0 \\
\hline ß1- Satisfação com o curso & 0,044775 & 0,01701 & 2,632341 & 0,0085 \\
\hline ß2- Satisfação com a IES & 0,004947 & 0,014887 & 0,332276 & 0,7397 \\
\hline$\beta 3$ - Oportunidades de desenvolvimento & $-0,00452$ & 0,010328 & $-0,43807$ & 0,6613 \\
\hline R-squared & 0,200496 & Mean dependent var & 6,674419 & \\
\hline Adjusted R-squared & 0,171246 & S.D. dependent var & 0,710292 & \\
\hline S.E. of regression & 0,646621 & Akaike info criterion & 2,329571 & \\
\hline Sum squared resid & 34,28572 & Schwarz criterion & 2,443727 & \\
\hline Log likelihood & $-96,1716$ & Hannan-Quinn criter. & 2,375514 & \\
\hline Restr. log likelihood & $-100,471$ & LR statistic & 8,598005 & \\
\hline Avg. log likelihood & $-1,11827$ & Prob(LR statistic) & 0,035142 & \\
\hline
\end{tabular}

Fonte: Dados da Pesquisa.

Em Vitória, o índice de 17\% da variação acerca da satisfação com a experiência acadêmica pode ser explicado a partir do fator satisfação com o curso e isso é demonstrado pelo coeficiente múltiplo de regressão $\left(\mathrm{R}^{2}\right)$ ajustado.

Pode-se inferir que há outros atributos, fora os relacionados, que podem ser considerados para entender melhor a relação entre a satisfação com a experiência acadêmica e a vontade de concluir o curso superior de Engenharia nessa IES da cidade de Vitória e isso é demonstrado pela existência de variância não explicada (residual) na variável dependente.

\section{Análise de regressão múltipla para a cidade de belo horizonte}

Também há evidências de relação positiva entre a satisfação com o curso e a vontade de concluir o curso de Engenharia para a cidade de Belo Horizonte. Os demais fatores - satisfação com a IES e oportunidades de desenvolvimento - também não são estaticamente significantes. Isso pode ser verificado pelos resultados entrados na Tabela 5, onde o regressor $\mathrm{b}_{1}(0,067291$; $\mathrm{p}$-value $=0,0001)$ representa a significância estatística.
Tabela 5: Modelo de Regressão para Belo Horizonte

\begin{tabular}{|c|c|c|c|}
\hline \multicolumn{4}{|c|}{ Dependent Variable: CONCLUSÃO DO CURSO } \\
\hline \multicolumn{4}{|c|}{ Method: QML - Normal Count (Quadratic hill climbing) } \\
\hline \multicolumn{4}{|c|}{ Included observations: 56} \\
\hline \multicolumn{4}{|l|}{ QML parameter used in estimation: 1} \\
\hline \multicolumn{4}{|c|}{ Convergence achieved after 4 iterations } \\
\hline \multicolumn{4}{|c|}{ QML (Huber/White) standard errors \& covariance } \\
\hline & Coefficient & Std. Error z-Statistic & Prob. \\
\hline$\beta 0$ & 1,536947 & $0,08723 \quad 17,61939$ & \\
\hline$\beta 1-$ Satisfação com o curso & 0,067291 & 3,876233 & 0,0001 \\
\hline ß2- Satisfação com a IES & 0,014236 & 0,016947 & 0,5756 \\
\hline B3- Oportunidades de desenvolvimento & $-0,01706$ & $-0,86946$ & 0,3481 \\
\hline R-squared & 0,254125 & Mean dependent var & 6,535714 \\
\hline Adjusted R-squared & 0,211094 & S.D. dependent var & 0,893701 \\
\hline S.E. of regression & 0,793789 & Akaike info criterion & 2,565828 \\
\hline Sum squared residuals & 32,76522 & Schwarz criterion & 2,710495 \\
\hline Log likelihood & $-67,8432$ & Hannan-Quinn criter. & 2,621915 \\
\hline Restr. log likelihood & $-73,4248$ & LR statistic & 11,16335 \\
\hline Avg. log likelihood & $-1,21149$ & Prob(LR statistic) & 0,010875 \\
\hline
\end{tabular}

Fonte: Dados da pesquisa

Em outras palavras, há evidências que os atributos mais valorizados pelos estudantes de Engenharia da IES privada da cidade de Belo Horizonte abrangem o fator satisfação com o curso. Ou seja, quanto mais satisfeitos os alunos estiverem com o curso, mais vontade eles terão de concluir o curso de Engenharia nessa cidade. $O$ desvio padrão de 0,01736 também é baixo contribuindo para mostrar a confiabilidade desse coeficiente de regressão.

Em Belo Horizonte, 21\% da variação acerca da satisfação com a experiência acadêmica pode ser explicada a partir do fator satisfação com o curso, dado pelo coeficiente múltiplo de regressão $\left(\mathrm{R}^{2}\right)$ ajustado.

Existem indícios de que outros atributos podem ser considerados para entender melhor a relação entre a satisfação com a experiência acadêmica e a vontade de concluir o curso superior de Engenharia nessa IES da cidade de Belo Horizonte. Esse resultado vem da existência de variância não explicada (residual) na variável dependente.

\section{Análise de regressão múltipla para ambas as cidades - vitória e belo horizonte}

Ao se analisar os resultados do modelo consolidado para ambas as cidades, mostrados na Tabela 6 , podese inferir que há evidências de relação positiva entre a satisfação com o curso e a vontade de concluir o curso de Engenharia em ambas as cidades, o que pode ser 
visto pelo regressor $\mathrm{b}_{1}(0,054894 ; \mathrm{p}$-value $=0,0000)$ que apresenta significância estatística. Os demais fatores - satisfação com a IES e oportunidades de desenvolvimento - também não são estaticamente significantes.

Em outras palavras, também há evidências que os atributos mais valorizados pelos estudantes de Engenharia da IES privada em ambas as cidades abrangem o fator satisfação com o curso. Ou seja, quanto mais satisfeitos os alunos estiverem com o curso, mais vontade eles terão de concluir o curso de Engenharia nas IES das cidades de Belo Horizonte e Vitória. O desvio padrão de 0,011897 é baixo contribuindo para mostrar a confiabilidade desse coeficiente de regressão consolidado para ambas as cidades.

Tabela 6: Modelo de regressão para ambas as cidades

\begin{tabular}{|c|c|c|c|c|}
\hline \multicolumn{5}{|c|}{ Dependent Variable: CONCLUSÃO DO CURSO } \\
\hline \multicolumn{5}{|c|}{ Method: QML - Normal Count (Quadratic hill climbing) } \\
\hline \multicolumn{5}{|c|}{ Included observations: 142} \\
\hline \multicolumn{5}{|l|}{ QML parameter used in estimation: 1} \\
\hline \multicolumn{5}{|l|}{ Convergence achieved after 4 iterations } \\
\hline \multicolumn{5}{|c|}{ QML (Huber/White) standard errors \& covariance } \\
\hline & Coefficient & Std. Error & z-Statistic & Prob. \\
\hline$\overline{B O}$ & 1,605521 & 0,06792 & 23,63835 & 0 \\
\hline$\beta 1$ - Satisfação com o curso & 0,054894 & 0,011897 & 4,613988 & 0 \\
\hline ß2-Satisfação com a IES & 0,006525 & 0,011656 & 0,559849 & 0,5756 \\
\hline$\beta 3-$ Oportunidades de desenvolvimento & $-0,00914$ & 0,009742 & $-0,93832$ & 0,3481 \\
\hline R-squared & 0,22397 & Mean dependent var & & 6,619718 \\
\hline Adjusted R-squared & 0,2071 & S.D. dependent var & & 0,787602 \\
\hline S.E. of regression & 0,70132 & Akaike info criterion & & 2,37221 \\
\hline Sum squared resid & 67,87529 & Schwarz criterion & & 2,455473 \\
\hline Log likelihood & $-164,427$ & Hannan-Quinn criter. & & 2,406045 \\
\hline Restr. log likelihood & $-174,222$ & LR statistic & & 19,5895 \\
\hline Avg. log likelihood & $-1,15794$ & Prob(LR statistic) & & 0,000206 \\
\hline
\end{tabular}

Fonte: Dados da pesquisa.

O coeficiente múltiplo de regressão $\left(R^{2}\right)$ ajustado indica que, em ambas as cidades, $20,7 \%$ da variação acerca da satisfação com a experiência acadêmica pode ser explicada a partir do fator satisfação com o curso.

Os resultados consolidam que há variância não explicada (residual) na variável dependente. Novamente, isso demonstra que há outros atributos que podem ser considerados para entender melhor essa relação entre a satisfação com a experiência acadêmica e a vontade de concluir o curso superior de Engenharia nas IES de Vitória e Belo Horizonte.
Os resultados revelam que há evidências que os atributos mais valorizados pelos estudantes de Engenharia das IES privadas das cidades de Vitória e Belo Horizonte abrangem o fator satisfação com o curso, que é composto por: corpo docente com domínio do conteúdo, estratégia de aula utilizada pelos professores, empregabilidade potencial do curso, interesse do corpo docente em atender os alunos durante a aula, compromisso da instituição de ensino com a qualidade da formação do aluno, avaliação proposta pelos professores, existência de equipamentos modernos, relacionamento com os professores, relevância do conteúdo das matérias, recursos e equipamentos disponíveis pela instituição, atendimento personalizado aos alunos e atendimento a reclamações. Esses achados, na sua maioria, estão em consonância com os resultados de alguns autores da área. A satisfação com corpo docente com domínio do conteúdo vai ao encontro dos resultados obtidos pela literatura, que descreve os estudantes como satisfeitos em relação ao domínio do conteúdo com os docentes possuidores de saberes sólidos das disciplinas ministradas (MILAN et al., 2014; GOMES; DAGOSTINI; CUNHA, 2014; ANTONELLI; COLAUTO; CUNHA, 2011).

A satisfação com a estratégia de aula utilizada pelos professores está coerente com o estudo de Antonelli, Colauto e Cunha (2011) caracterizada como o poder de ser mutável frente aos obstáculos nos processos. A pesquisa dos autores também teve resultados satisfatórios para a variável interesse do corpo docente em atender os alunos durante a aula. Cruz (2014) concluiu que a maioria de respondentes alegaram estarem satisfeitos com os professores que respondem prontamente as questões de seus discentes em qualquer momento da aula.

Souza e Reneirt (2010) obtiveram resultados semelhantes ao dessa pesquisa, ao apresentar indicadores que demonstram satisfação com a empregabilidade potencial do curso e avaliação proposta pelos professores.

O compromisso da instituição de ensino com a qualidade da formação do aluno, compreende as ações necessárias com o intuito de melhorar o processo de aprendizagem de uma maneira geral. E conforme dados da pesquisa de Antonelli, Colauto e Cunha (2011), os estudantes também se mostraram satisfeitos. 
Souza Junior, Oliveira e Canuto (2012) afirmam que um indicador que exige atenção, é a satisfação com a existência de equipamentos modernos. Os autores ainda salientam que a falta de equipamentos modernos dificulta a relação entre teoria e prática e compromete a atratividade das aulas.

A forma pelo qual os professores ministram o conteúdo das disciplinas para os alunos, provas e atividades constituem um fator organização do curso de Gomes Dagostini e Cunha (2013), que avalia como os professores se relacionam com os discentes e como as disciplinas estão relacionadas. Desta forma, os estudantes se mostram satisfeitos em sua maioria com o relacionamento com os professores.

Souza e Reinert (2010) afirmam que a satisfação com a relevância do conteúdo das matérias se torna mais crítica com o decorrer da experiência acadêmica do estudante, uma vez que ele passa a ter mais envolvimento com o processo de aprendizagem.

Enfatizar as formas como os docentes se interagem com os discentes de forma a facilitar o aprendizado, com um atendimento personalizado aos alunos, e a atenção dada aos alunos em sala de aula destacou índices altos no estudo de Gomes, Dagostini e Cunha (2013).

A satisfação com o atendimento a reclamações é um dos atributos mais valorizados pelos estudantes. Souza Junior, Oliveira e Canuto (2012) mostraram em sua pesquisa que apenas 3\% dos estudantes relataram insatisfação com o atendimento a reclamações. O tratamento de reclamações, segundo Cruz (2014), é um indicador que merece atenção, uma vez que os alunos mais insatisfeitos têm a tendência de reclamar mais.

Embora possa haver diferenças nos contextos social e econômico dessas cidades, esses resultados mostram evidências similares para as IES dessas capitais. Essa similaridade de evidências, contudo, não significa que não há necessidade das IES aprofundarem as pesquisas de marketing sobre o comportamento desses alunos. Até porque os demais fatores - satisfação com a IES e as oportunidades de desenvolvimento - não são estaticamente significantes. Os resultados mostram que é importante conhecer porque tais fatores não são significantes. Além disso, essas IES poderiam usar o marketing educacional para criarem ações de marketing que vão além da formulação de estratégias de captação de alunos para que o curso tenha viabilidade econômica ou de mídia e promoções (NUNES et al., 2008). Essas ações têm que estar voltadas para incentivar os alunos a concluir o curso superior.

\section{Considerações Finais}

No presente estudo, foi desenvolvido um questionário adaptado a partir da escala de Schleich, Polydoro e Santos (2006) que articula elementos de satisfação com o curso, oportunidade de desenvolvimento e satisfação com a Instituição para verificar a satisfação dos estudantes de ensino superior com a experiência acadêmica e a sua relação com a vontade de concluir o curso.

Os dados do questionário serviram de base para as regressões lineares rodadas. Os resultados corroboram parcialmente com a literatura existente (MILAN et al., 2014; GOMES; DAGOSTINI; CUNHA, 2014; ANTONELLI; COLAUTO; CUNHA, 2011) evidenciando que os atributos que explicam o fator satisfação com o curso são os mais valorizados pelos estudantes, em ambas as cidades, para medir a satisfação com a experiência acadêmica. E, por conseguinte, influenciam a vontade de concluir o curso superior de Engenharia em ambas as cidades do estudo. Ou seja, a vontade dos alunos de concluir o curso superior nas respectivas IES muda de forma moderada, porém, coerente e sistematicamente na medida em que muda a percepção dos alunos com a satisfação com tais atributos.

Outro fator importante diz respeito aos modelos, que mostraram que cerca de $20 \%$ da satisfação com a experiência acadêmica pode ser explicada a partir do fator satisfação com o curso. Ou seja, ainda há variância não explicada sobre a essa relação entre a satisfação com a experiência acadêmica e a vontade de concluir o curso superior de Engenharia. Podese inferir com isso que ainda há outros atributos que podem ser considerados para entender melhor essa relação. Nessa pesquisa, em particular, foram utilizados 28 atributos.

Uma limitação do presente estudo e, talvez a principal, é que os resultados dessa pesquisa não podem ser generalizados, uma vez as duas amostras foram não 
probabilísticas. Além disso, a coleta de dados foi feita com alunos do primeiro e segundo anos, o que pode causar um viés na amostra, pois quem está no primeiro período não está disposto a abandonar ainda. Além do mais, não tem condições de dizer se está insatisfeito com o curso, pois é muito cedo. A insatisfação tende a ocorrer mais tarde, após o desenvolvimento de capacidade de avaliação crítica do aluno sobre o curso. A partir dessas declarações os estudos posteriores poderiam utilizar uma amostra significativa e a coleta de dados ser feita apenas com alunos nos últimos anos e poderiam se concentrar também na identificação de estratégias sobre o marketing educacional das IES, pois a sobrevivência dessas instituições, diante um mercado altamente competitivo, passa pelas suas habilidades e capacidades estratégicas para alcançarem bons resultados. Também sugere-se a realização dessa pesquisa em outras capitais brasileiras a fim de realizar comparação entre elas, além de pesquisas que abordem outros atributos de satisfação, além daqueles já discutidos na literatura.

\section{Referências}

AITKEN, Norman D. College Student Performance, Satisfaction and Retention: specification and estimation of a structural model source. The Journal of Higher Education, v. 53, n.1, p. 32-50, jan./feb. 1982.

ALVES, Helena; MAINARDES, Emerson Wagner; RAPOSO, Marlo. O Marketing no Ensino Superior: comparativo Brasil-Portugal. Revista de Administração FACES Journal Belo Horizonte, v. 10, n. 4, p. 35-64, set./dez. 2010.

ANDRADE, P.R; ARAUJO, H.C.R . Análise do Perfil dos Estudantes de uma IES: o marketing como ferramenta para as instituições de ensino superior. Brazilian Business Review, v.8, n.1, p.61-73, 2011.

ANTONIELLI, R.A.; COLAUTO, R.D.; CUNHA, J.V.A. Expectativas e satisfação dos alunos de ciências contábeis com relação as competências docentes.

Revista Ibero americana sobre qualidade, eficácia e mudança em educação, v.10, n. 1, p. 75-91, 2012.
BARBOSA, P. V.; MEZZOMO, F.; LODER, L. L. Motivos de Evasão no Curso de Engenharia Elétrica: realidade e perspectivas. In: Congresso Brasileiro de Educação em Engenharia, 39., 2011. Blumenau, SC. Anais... Blumenau, SC: ABENGE, 2011.

BARDAGI, M. P.; LASSANCE, M. C. P.; PARADISO, A C. Trajetória Acadêmica e Satisfação com a Escolha Profissional de Universitários em Meio de Curso. Revista Brasileira de Orientação

Profissional, v.4, n.1, p.153-166, 2003.

BEBER, S. J. N. Estado Atual dos Estudos sobre a Satisfação do Consumidor. In: Encontro da Associação Nacional dos Estudantes de PósGraduação em Administração, 1999. Foz do Iguaçu. Anais... Rio de Janeiro: ANPAD, 1999.

BENJAMIN, Michael; HOLLINGS Ann. Student Satisfaction: test of an ecological model. Journal of College Development, v.38, n. 3, p. 213-228, 1997.

BERGAMO, F. V. M.; GIULIANI, A. C.; GALLI, L. C. L. A. Modelo de Lealdade e Retenção de Alunos para Instituições do Ensino Superior: um estudo teórico com base no marketing de relacionamento. Brazilian Business Review, v. 8, n. 2, p. 43-66, 2011.

CARO, E. M.; BOLARÍN, F.C. Factors Affecting Students' Satisfaction in Engineering Disciplines: traditional vs. blended approaches. European Journal of engineering education, v.36, n.5, p.473483, out. 2011.

CARVALHO, F. A.; LEITE, V. F. Refinando a Conjetura PBZ: uma revisão da relação entre importância e tolerância em qualidade de serviços.

Revista de Administração Contemporânea, v.5, n.1, p. 43-60, jan./abr. 2008.

CLAYSON, D. E.; HALEY, D. A. Student Evaluations in Marketing: what is actually being measured? Journal of Marketing Education, v. 12, n. 3, p. 9-17, 1990.

CRUZ, A. C. O papel da Estratégia de Marketing de uma Instituição de Ensino 
Superior na Decisão de Escolha e Satisfação dos Estudantes: estudo de caso da faculdade de economia e gestão da UNTL. 2014. 180f. Dissertação (Mestrado em Marketing e Estratégia) Universidade do Minho, 2014.

FREITAS, A. A.; DORNELLAS, V. D.; BELHOT, V. R. Requisitos Profissionais dos Estudantes de Engenharia de Produção: uma visão através dos estilos de aprendizagem. In: Simpósio de Engenharia de Produção, 12., abr. 2006. São Paulo. Anais... São Paulo: UNESP, abr. 2006.

GOMES, G.; DAGOSTINI, L.; CUNHA, P. R. Satisfação dos Estudantes do Curso de Ciências Contábeis: estudo em uma faculdade do Paraná. Revista de Administração e Economia, v.4, n.2, p.102-123, 2013.

HAIR JR., J. F.; ANDERSON, R. E.; TATHAM, R. L.; BLACK, W. C. Análise Multivariada de Dados.

5. ed. Porto Alegre: Bookman, 2005.

HIDES, M. T.; DAVIES, J. J; JACKSON, S. Implementation of EFQM Excellence Model Self Assessment in the UK Higher Education Sector: lessons learned from other sectors. The TQM Magazine, v. 16, n. 3, p.194-201, nov. 2004.

LINS, L. M.; SALERNO, M. S.; ARAUJO, B. C.; GOMES, L. A. V.; NASCIMENTO, P. A. M. M.; TOLEDO, D. Escassez de Engenheiros no Brasil. Centro brasileiro de análise e planejamento, n.98, p.43-67, 2014.

MAINARDES, Emerson Wagner; DOMINGUES; Maria José Carvalho de Souza. Satisfação de Estudantes em Administração de Joinville/SC.

Revista Pensamento Contemporâneo em Administração, v. 4, n. 2, p. 76-94, mai./ago, 2010.

MILAN, G. S.; LARENTIS, F.; CORSO, A.; EBERLE, L.; LAZZARI, F.; DEONIR, T. Atributos de Qualidade dos Serviços Prestados por Uma IES e os Fatores que Impactam na Satisfação dos Alunos do Curso de Graduação em Administração. In: Encontro da Associação Nacional dos Estudantes de Pós-Graduação em Administração, 35., 2014. Rio de
Janeiro. Anais... Rio de Janeiro: ANPAD, 2014.

NATH, L.; ANDERSON, L. The Effects of a Classroom Discussion Technique on Student Satisfaction: a quasi-experiment. Mountain Rise International Journal of the Scholarship of Teaching and Learning, v.4, n.1, 2007.

NUNES, G. T.; LANZER, E. A.; SERRA, F. R.; FERREIRA, A.R.; MANUEL P. Emergência do Marketing nas Instituições de Ensino Superior: um estudo exploratório. Análise Porto Alegre, v.19, n.1, p. 173-198, jan./jun. 2008.

PASQUINI, Elaine Silvia; SANTOS, Marcelo dos; PASCUAL, Jose Valentim Iglesias; SUPPION, Klaus; Elmo Tambosi Filho. Avaliação dos Cursos de Ensino Superior através da Ótica Discente de Satisfação/Insatisfação. Revista da Faculdade de Administração e Economia, v.3, n. 2, p.147-165, 2012.

PASWAN, A. K.; YOUNG, J. A. Student Evaluation of Instructor: a nomelogical investigation using struuctural equation modeling. Journal of Marketing Education, v. 24, n. 3, p. 193-202, 2002.

PINHEIRO, M. R. C. A Percepção de Suporte Social da Família e dos Amigos como Elementos Facilitadores de Transição para o Ensino Superior. In: Congresso Galaico-Português de Psicopedagogia, 8., 2005. p. 467-485.

QUEVEDO-SILVA, Filipe; LIMA-FILHO, OLIVEIRA, Dario de; SAUER, Leandro; REINERT, José Nilson. Fatores Discriminantes no Grau de Satisfação de Estudantes de Administração. Revista de Economia e Administração, v.11, n.1, p. 28-45, jan./mar. 2012.

SARRICO, Cláudia S.; ROSA, Maria J. Student Satisfaction wich Portuguese Higher Institutions: the view diferente types of students. Tertiary Education and Management, v. 20, n. 2, p. 165178, 2014.

SCHLEICH, A. R. L.; POLYDORO, S. A. J.; SANTOS, A. A. A. Escala de Satisfação com a 
Experiência Acadêmica de Estudantes de Ensino Superior. Avaliação Psicológica, v.5, p.11-20, 2006.

SCHREINER, L. A. Linking Student Satisfaction and Retention. Research study: Azusa Pacific University, 2009.

SILVA, F. Q.; FILHO, D. O.L.; SAUER, L.;

REINERT J. N. Fatores Discriminantes no Grau de Satisfação de Estudantes de Administração. Revista de Economia e Administração, v.11, n. 1, p. 28-45, jan./mar. 2012.

SOUZA JUNIOR, Carlos Menezes; OLIVEIRA, Herbet A.; CANUTO, Römulo Augusto. O trabalho Pedagógico do Campus Estância do Instituto Federal de Sergipe, os Resultados Obtidos e as Relações destes com a Satisfação dos Discentes. In: Colóquio Internacional: Educação e Contemporaneidade, 6., 2012. Sergipe. Anais... Sergipe: GPECPOP, 2012.

SOUZA, S. A.; ALVES, F. M. S.; BUSS, R. N.; Satisfação de Estudantes do Curso de Graduação em Administração na Universidade Federal de Mato Grosso do Sul. In: Encontro da Associação Nacional de Pós-Graduação e Pesquisa em Administração, 32., 2008. Rio de Janeiro. Anais... Rio de Janeiro: ANPAD, 2008.

; REINERT, J. N. Avaliação de um Curso de Ensino Superior Através da Satisfação/ Insatisfação Discente. Avaliação, v. 15, n. 1, p. 159-176, mar. 2010.

TONTINI, G.; WALTER, S. A.; Antecedentes da Qualidade Percebida de um Curso de Administração: uma abordagem não linear. Revista Brasileira de

Gestão de Negócios, v. 13, n.40, p.264-280, jul./set. 2011.

VENTURINI, J.; PEREIRA, B.A.D.; VIEIRA, K.M.; MILACH, F. Satisfação dos Alunos do Curso de Ciências Contábeis da UNIFRA: um estudo à luz das equações estruturais. In: Congresso USP Controladoria e Contabilidade, 8., 2008. Anais... USP, 2008. 